\title{
THE FRÉCHET SPACE $\omega$ ADMITS A STRICTLY STRONGER SEPARABLE AND QUASICOMPLETE LOCALLY CONVEX TOPOLOGY
}

\author{
SUSANNE DIEROLF
}

Let $\mathfrak{L}$ denote the class of all locally convex Hausdorff spaces $(E, \mathfrak{Z})$ with the following property: Every locally convex Hausdorff topology $\subseteq \subset \mathfrak{I}$ on $E$ has the same subfamily summable sequences as $\mathfrak{T}$. Several articles have been devoted to the investigation of the richness of $\mathfrak{Z}$, e.g., Kalton [4], Labuda [6], [7], Graves [3]; see also the references in [3]. For example, $\mathfrak{L}$ contains every fully complete locally convex space which does not contain $1^{\infty}[6$, p. $219,(8)]$, hence every separable Fréchet space. E. Thomas asked in a letter of 1976 whether $\mathfrak{L}$ even contains every separable quasicomplete space. This note provides a negative answer to this question.

We will use the following results about separability which we prove for general topological vector spaces.

LEMMA. Every finite codimensional linear subspace $H$ in a separable topological vector space $E$ is separable.

Proof. We may at once assume that $H=\operatorname{ker} f$, where $f$ is a discontinuous linear form on $E$.

$E$ contains a dense linear subspace $L$ of countable dimension. For every $x \in E$ let $L_{x}$ denote the linear span of $L \cup\{x\}$. We denote the topology of $E$ by $\mathfrak{T}$. The strongest linear topology $\subseteq$ on $E$ such that for every $x \in E$, the relative topologies $\mathfrak{S} \mid L_{x}$ and $\mathfrak{I} \mid L_{x}$ coincide, is clearly stronger than $\mathfrak{T}$. Moreover $\mathfrak{S}|L=\mathfrak{T}| L$ and $L$ is dense in $(E, \subseteq)$, hence $\mathfrak{I}=\subseteq$ by [2, p. 349, Lemma 1]. Since $f$ is discontinuous we deduce that for some $z \in E$ the restriction $f \mid L_{z}$ is discontinuous, whence $H \cap L_{z}$ is dense in $L_{z}$. Thus $H \cap L_{z}$ is dense in $E$ and hence dense in $H$. Since $H \cap L_{z}$ is of countable dimension, we have proved that $H$ is separable.

(For a locally convex space $E$, a somewhat technical proof of the lemma has been given by Valdivia in [8, p. 195, Lemma 2].)

Proposition. Let $(E, \mathfrak{I})$ be a separable topological vector space over $\mathbf{K} \in\{\mathbf{R}, \mathbf{C}\}$ and let $\left(f_{n}\right)_{n \in N}$ be a sequence of linear forms on $E$. Then the initial topology $\mathscr{F}$ on $E$ with respect to the identity map id: $E \rightarrow(E, \mathfrak{T})$ and all the functionals $f_{n}: E \rightarrow \mathbf{K}$ $(n \in N)$ is again separable.

Received by the editors September 29, 1980.

1980 Mathematics Subject Classification. Primary 46A35, 46A05.

Key words and phrases. Separable topological vector spaces, subseries convergent series, Orlicz-Pettis property. 
Proof. For every $n \in \mathbf{N}$, the space $E$ provided with the initial topology $\mathscr{F}_{n}$ with respect to id: $E \rightarrow(E, \mathfrak{T})$ and $f_{i}: E \rightarrow \mathbf{K}(1<i<n)$, is the topological direct sum of $\left(\cap_{1<i<n} \operatorname{ker} f_{i}, \mathfrak{I} \mid \cap_{1<i<n} \operatorname{ker} f_{i}\right)$ and a finite dimensional linear subspace, hence separable according to the lemma. Since $\mathscr{F}_{n} \subset \mathscr{F}_{n+1}(n \in N)$ and $\mathscr{F}$ equals the supremum $\bigvee_{n \in N} \mathscr{F}_{n}$, we obtain the separability of $(E, \mathscr{F})$.

The separable Fréchet space $\omega:=\mathbf{K}^{\mathbf{N}}$ provided with the product topology $\mathfrak{B}$, clearly carries the initial topology with respect to the sequence of linear forms $p_{n}$ : $\omega \rightarrow \mathbf{K},\left(x_{m}\right)_{m \in N} \mapsto x_{n},(n \in N)$. Thus we get the following:

COROLlaRY. For every separable linear topology $\mathfrak{I}$ on $\omega$ the supremum $\mathfrak{I} \vee \mathfrak{B}$ is again separable.

REMARK. We mention that the supremum of two separable linear topologies need not be separable. In fact, let $(E, \mathfrak{I})$ be a separable locally convex space containing a nonseparable linear subspace $L$. Choose a linear subspace $M \subset E$ such that $L \cap M=\{0\}$ and $L+M=E$. Then the initial topology $\subseteq$ on $E$ with respect to $j$ : $E \rightarrow(E, \mathfrak{I}), j(x+y):=x-y(x \in L, y \in M)$ is also separable. One verifies without difficulty that $(E, \mathfrak{I} \vee \mathfrak{S})$ is the topologically direct sum of $(L, \mathfrak{T} \mid L)$ and $(M, \mathfrak{I} \mid M)$, hence not separable.

EXAMPLE. We consider the noncomplete separable Montel space $X$ constructed by Amemyia, Kōmura [1] (cf. also Knowles, Cook [5]), whose dimension is not less than the dimension of $\omega$ and in which every bounded subset has a finite dimensional linear span (see [1], [5]). Consequently there exists an injective linear map $f$ : $\omega \rightarrow X$ with separable range. Let $\mathfrak{I}$ denote the initial topology on $\omega$ with respect to $f: \omega \rightarrow X$, which is clearly locally convex.

On account of the corollary, $(\omega, \mathfrak{I} \vee \mathfrak{B})$ is separable. Moreover, every bounded set in $(\omega, \mathfrak{I} \vee \mathfrak{B})$ has finite dimensional linear span, whence in particular, $(\omega, \mathfrak{I} \vee$ $\mathfrak{B})$ is quasicomplete.

Finally, the sequence $\left(e_{n}\right)_{n \in N}$ of unit vectors $e_{n}=\left(\delta_{n m}\right)_{m \in N} \in \omega$ is subfamily summable in $(\omega, \mathfrak{B})$, but not bounded, hence not summable, in $(\omega, \mathfrak{I} \vee \mathfrak{P})$. Thus $(\omega, \mathfrak{I} \vee \mathfrak{B}) \notin \mathfrak{R}$.

\section{REFERENCES}

1. I. Amemyia and Y. Kōmura, Über nicht vollständige Montelräume, Math. Ann. 177 (1968), 273-277.

2. S. Dierolf and U. Schwanengel, Examples of locally compact non-compact minimal topological groups, Pacific J. Math. 82 (1979), 349-355.

3. W. H. Graves, Universal Lusin measurability and subfamily summable families in abelian topological groups, Proc. Amer. Math. Soc. 73 (1979), 45-50.

4. N. J. Kalton, Subseries convergence in topological groups and vector spaces, Israel J. Math. 10 (1971), $402-412$.

5. R. J. Knowles and T. A. Cook, Incomplete reflexive spaces without Schauder bases, Proc. Cambridge Philos. Soc. 74 (1973), 83-86.

6. I. Labuda, A note on exhaustive measures, Ann. Soc. Math. Polon. Ser. I Comment. Math. Prace Mat. 18 (1975), 217-221.

7. Universal measurability and summable families in tos, Indag. Math. 41 (1979), 27-34.

8. M. Valdivia, On quasi-completeness and sequential completeness in locally convex spaces, J. Reine Angew. Math. 276 (1975), 190-199.

Mathematisches Institut der Universttït MÜnchen, D-8000 MÜnchen 2, West Germany 